\title{
Impact of Mycophenolate Mofetil on Wound Complications and Lymphoceles after Kidney Transplantation
}

\author{
Kai Lopau ${ }^{\mathrm{a}}$ Karin Syamken ${ }^{\mathrm{a}}$ Peter Rubenwolf ${ }^{\mathrm{b}}$ Hubertus Riedmiller $^{\mathrm{b}}$ \\ Christoph Wanner ${ }^{a}$ \\ ${ }^{a}$ Department of Internal Medicine I, Division of Nephrology, and ${ }^{b}$ Department of Urology, \\ University of Wuerzburg, Wuerzburg, Germany
}

\section{Key Words}

Kidney transplantation - Mycophenolate mofetil • Immunosuppression · Wound healing $\cdot$ Lymphoceles

\begin{abstract}
Background/Aims: Despite improved efficacy, modern immunosuppressive agents may show unanticipated side effects. In this study we investigated the possible interactions of mycophenolate mofetil (MMF) with wound healing and lymphocele formation. Methods: We conducted a retrospective single-center analysis in 144 patients receiving a cyclosporine A-based immunosuppression with prednisolone and either MMF $(n=77)$ or azathioprine (AZA, $n=77)$. Endpoints were incidences of lymphocele formation and nonprimary wound healing during 6 months' follow-up. Results: AZA-treated patients had more rejection episodes and consecutively more steroid pulses, both being potential risk factors for endpoints. No graft was lost in any group and graft function was comparable. AZA patients demonstrated a trend for more frequent wound infections. Fluid accumulation around the graft, however, was more frequent in the MMF group ( $O R=2.6 ; p=0.03)$. Consequently, more drainage maneuvers (17 vs. 5 interventions) and sclerotherapies (8 vs. 0 interventions) were undertaken in MMF patients. Pre-
\end{abstract}

assigned risk factors for lymphoceles reported before did not differ between both cohorts; patients experiencing acute rejection episodes had even less symptomatic lymphoceles $(n=23)$. Conclusion: We found a possible relationship between the administration of MMF and lymphocele formation. To avoid the hazard of reinterventions, the prolongation of hospitalization and impairment of graft function, it requires awareness and attention in patients treated with this immunosuppressant.

Copyright $\odot 2010$ S. Karger AG, Basel

\section{Introduction}

Modern immunosuppressive combinations allow 1-year graft survival coming close to 100 percent with extraordinarily low rejection rates after kidney transplantation [1]. However, also the newer immunosuppressive agents like sirolimus, everolimus and both mycophenol acid derivates are not free from partly disabling side effects [2]. Wound healing disorders as well as lymphoceles have been reported as potentially serious postoperative problems in up to $20 \%$ of all kidney transplant (KTx) recipients [3]. Wound healing disorders predispose for infectious complications in the most vulnerable period

\section{KARGER}

Fax +41613061234 E-Mail karger@karger.ch www.karger.com
(C) 2010 S. Karger AG, Base

$1420-4096 / 10 / 0331-0052 \$ 26.00 / 0$

Accessible online at:

www.karger.com/kbr
Kai Lopau, MD

University of Wuerzburg, Department of Internal Medicine I

Division of Nephrology, Center of Internal Medicine (ZIM)

Oberduerrbacher Strasse 6, DE-97080 Wuerzburg (Germany)

Tel. +49931 201 1, Fax +49931 201639 305, E-Mail lopau_k@klinik.uni-wuerzburg.de 
with relatively high immunosuppression [4]. Lymphoceles can lead to obstruction of the graft ureter as well as the graft vasculature and therefore to acute and chronic functional impairment [5]. In addition, even a distinct fluid accumulation near to the wound predisposes for secondary infection [6].

Humar et al. [7] disclosed a trend for an increasing rate of wound complications with contemporary immunosuppressive treatment. In our own transplant population we also had the impression that the incidence of this type of postoperative complications increased over the past years. Therefore, this retrospective analysis was done to investigate if a modern immunosuppressive triple therapy based on cyclosporine A (CsA) and mycophenolate mofetil (MMF) together with steroids differs from our previous regimen with CsA, azathioprine (AZA) and steroids in respect to wound complications during the perioperative period until 6 months after transplantation. Multivariate analysis was conducted to search for other potential causes for this type of postoperative disorders.

\section{Patients and Methods}

\section{Patient Selection}

In this single-center retrospective analysis we compared two cohorts of 77 patients each transplanted between May 1990 and May 2003 who received either maintenance immunosuppressive treatment with CsA and prednisolone and either mycophenolate mofetil (MMF group) or azathioprine (AZA group). Patients in the AZA cohort were transplanted in the era between 1990 and 2001, MMF patients received their graft between 1995 and 2003. The principal inclusion criterion for this evaluation was a technically successful kidney transplantation with graft survival for at least 6 months. Patients who received both living-related and living-unrelated grafts were included. Both cohorts were followed up for 6 months after KTx. The groups differed in the time point of transplantation: more AZA patients were transplanted in the years before 1997, more MMF patients thereafter. Patients were matched for sex, age, concomitant immunosuppressive therapy and extrapolated prednisolone dosages.

\section{Endpoints and Definitions}

Endpoints of this analysis were the incidence of wound healing difficulties, lymphoceles and the need for surgical reintervention. Wounds were inspected daily by experienced nurses until discharge, thereafter in case of delayed wound closure or infection at least every other day in the outpatient department. Ultrasound examination was done daily until postoperative day 7 (PO-d7), thereafter every other day until week 3 and then weekly until the end of month 2 or out of schedule in case of any abnormalities. All ultrasound results were done or confirmed by one experienced ultrasound examiner (K.L.). Two ultrasound machines were used (Siemens Sonoline Elegra until 1995, Siemens Sonoline Versa Pro thereafter; Siemens AG, Erlangen, Germany).
We defined 'wound healing difficulty' as every wound not healing primarily or wounds with nonadapted wound edges after removal of suture material or wounds with prolonged fluid drainage, local infection or apparent cellulitis. We defined 'lymphocele' as any distinct fluid collection around the graft, as 'symptomatic lymphocele' fluid collections causing compression of the graft ureter or the graft or iliac vessels, thrombosis of graft vessels or iliac vessels, abdominal pain or discomfort or transplant malfunction not being explained by other causes.

\section{Immunosuppression}

Induction therapy with the interleukin-2 receptor antagonist basiliximab was administered to $5.1 \%$ in the AZA group and $53.2 \%$ in the MMF group. All patients thereafter received triple maintenance immunosuppression. Standard treatment consisted of CsA and steroids together with a proliferation blocking agent AZA or MMF. The initial dose of steroids consisted of $250 \mathrm{mg}$ methylprednisolone, tapered to $10 \mathrm{mg}$ of prednisolone after 3 months and $5 \mathrm{mg}$ after 6 months. CsA dosages were tapered according to $\mathrm{C}_{0}$ levels (CsA: $150-250 \mathrm{ng} / \mathrm{ml}$ in months $1-3$, thereafter 120-200 ng/ml). In cases of presumed or biopsy-proven rejection, bolus therapy with methylprednisolone $(3 \times 250 \mathrm{mg}$ for 3 days followed by $3 \times 125 \mathrm{mg}$ for 3 days) was administered. In some cases patients received polyclonal or monoclonal antibodies for antirejection therapy (table 1).

\section{Clinical and Laboratory Data}

All patients gave written informed consent for the evaluation of their records. Data handling was conducted in accordance with the Helsinki Declaration of the World Medical Association. Patient's complete hospital and outpatient records were reviewed. As baseline characteristics were recorded age, sex, date of transplant, cause of end-stage renal disease, dialysis modality and time on dialysis and history of diabetes. During the observation period of 6 months we assessed graft function expressed as serum creatinine, dosages of the different immunosuppressive agents, body weight and BMI in bimonthly intervals. Immunosuppression was recorded at every specific time point as CsA trough level and actual steroid dose in $\mathrm{mg} / \mathrm{kg} \times$ day. CsA-C2 levels have not been determined. Furthermore, the incidences of post-transplant diabetes, wound healing difficulties, lymphoceles and the type of treatment as well as acute rejections were recorded. The study was approved by the local institutional review board.

\section{Statistics}

For statistical analysis, the Statistical Package for Social Sciences (SPSS for Windows V15.0; SPSS GmbH, Munich, Germany) was used. Quantitative data were expressed as mean \pm SEM. In a first step all variables were tested for normal distribution with the Kolmogorov-Smirnov test. Non-normally distributed variables were compared with the Mann-Whitney U test, normally distributed variables with the paired and unpaired Student's t test. Thereafter, we performed multiple univariate and multivariate logistic regression analyses for variables considered most likely linked to the predefined endpoints. These were recipient age at transplant, gender, diabetes status before KTx, BMI before KTx, post-transplant diabetes mellitus (PTDM), induction therapy, acute rejection prevalence, serum creatinine at different observation points and therapy with MMF versus AZA. $\mathrm{p}<0.05$ was considered statistically significant. 
Table 1. Baseline characteristics of the patients

\begin{tabular}{lllc}
\hline & $\begin{array}{l}\text { MMF group } \\
(\mathrm{n}=77)\end{array}$ & $\begin{array}{l}\text { AZA group } \\
(\mathrm{n}=77)\end{array}$ & $\mathrm{p}$ \\
\hline Age, years & $54.6 \pm 12.9$ & $48.6 \pm 12.0$ & 0.40 \\
Gender, M/F & $44 / 33$ & $50 / 27$ & 0.33 \\
Time on dialysis, months & $50.1 \pm 37.1$ & $44.2 \pm 28.1$ & 0.26 \\
Dialysis modality, HD/PD & $59 / 17$ & $57 / 19$ & 0.43 \\
Number of grafts, 1st/2nd & $72 / 5$ & $71 / 6$ & 0.50 \\
Patients with PRA >20\%/<20\% & $70 / 7$ & $73 / 4$ & 0.42 \\
Mean PRAs, \% & $4.1 \pm 15.1$ & $2.7 \pm 11.3$ & 0.49 \\
Mode of KTx, DDO/LDO & $67 / 10$ & $74 / 3$ & 0.14 \\
Diabetes before KTx, n (\%) & $7(9.1)$ & $13(16.9)$ & 0.19 \\
PTDM, n (\%) & $18(23.4)$ & $19(24.1)$ & 0.30 \\
BMI before KTx & $24.7 \pm 4.4$ & $24.1 \pm 3.7$ & 0.36 \\
Anti-IL2-R induction therapy, n (\%) & $41(53.2)$ & $4(5.1)$ & $<0.001$ \\
ARE, n (\%) & $25(32.5)$ & $45(58.4)$ & 0.009 \\
Prednisolone pulses, n (\%) & $22(28.6)$ & $45(58.4)$ & 0.003 \\
Antirejection therapy with ATG/OKT 3 & $1 / 3$ & $2 / 4$ & 0.24 \\
\hline
\end{tabular}

PRA = Panel reactive antibodies DDO = deceased donor organ $\mathrm{LDO}=$ living donated organ; $\mathrm{ARE}=$ suspected or biopsy-proven acute rejection episode; $\mathrm{ATG}=$ antithymocyte globulin.

\section{Results}

\section{Baseline Parameters}

The two groups showed no statistical differences concerning gender distribution, dialysis modality, duration of dialysis treatment before transplantation, mode of transplantation and graft number. There was a nonsignificant trend for older recipient age combined with longer dialysis vintage in the MMF group, most likely a result of a later transplant era of this group. A detailed description of the baseline parameters of both cohorts is presented in table 1.

\section{Maintenance Immunosuppression}

Both study groups received a triple maintenance therapy based on CsA. There was a trend for higher CsA trough levels in the MMF group without significant differences (table 2). C2 levels or AUCs were not measured routinely. Steroid dosages were comparable between both groups. Our immunosuppressive regimen aimed for $1 \mathrm{mg} / \mathrm{kg} \times$ day AZA or a fixed dose of 2,000 mg MMF/ day, respectively, in order to achieve a sufficient inhibition of lymphocyte proliferation. However, neither AZA nor MMF were dosed as aimed for. Full-dose MMF was given in only $66.8 \%$ of all patients at month 2 , in $64.9 \%$ of all patients at month 4 and in $55.8 \%$ of all patients at month 6. This was in nearly all cases due to side effects, especially gastrointestinal complaints or, less often, marked myelosuppression. MMF trough levels were determined at that time; however, we could not demonstrate any statistical relationship of drug levels with side effects (data not shown).

\section{Wound Complications and Fluid Collections}

Wound infections and local healing difficulties were observed more often in the AZA group ( $n=13,17 \%$ vs. $\mathrm{n}=8,10 \%$ in the MMF group, $\mathrm{p}=0.24$ ), however these outcomes were not significantly different. Six episodes in the AZA group as well as six in the MMF group could be handled conservatively, in 7 (AZA) compared to 2 cases (MMF) surgical interventions had to be performed ( $\mathrm{p}=$ 0.09). None of the patients needed more than one intervention for definite wound closure.

Fluid collections around the graft were demonstrated sonographically in 46 patients receiving MMF (60\%) versus 31 patients receiving AZA (40\%); this difference was significantly different $(\mathrm{p}=0.016)$. Effusions were first detected $18 \pm 3$ days (AZA) versus $17 \pm 5$ days (MMF) after KTx. Before the detection of any effusion, drains have been in situ for $8 \pm 2$ days (AZA) and $8 \pm 3$ days (MMF), respectively. Symptomatic lymphoceles, most of them leading to abdominal discomfort or being located near the graft vessels, were observed in $15 \mathrm{MMF}$ patients versus 8 AZA patients (19 vs. 10\%, n.s.). Altogether, this led to 17 (MMF) versus 5 (AZA) ultrasound-guided drainage procedures (4 patients in the MMF group vs. no 
Table 2. Maintenance immunosuppressive therapy (mean \pm SD)

\begin{tabular}{lcc}
\hline & MMF group & AZA group \\
\hline CsA trough level, ng/ml & & \\
$\quad$ Month 2 & $168.6 \pm 45.5$ & $137.6 \pm 47.6$ \\
$\quad$ Month 4 & $154.8 \pm 66.7$ & $131.8 \pm 39.6$ \\
$\quad$ Month 6 & $134.9 \pm 43.2$ & $122.2 \pm 40.5$ \\
Prednisolone dosage, mg/day & & \\
$\quad$ Month 2 & $18.1 \pm 8.1$ & $17.8 \pm 6.3$ \\
$\quad$ Month 4 & $9.0 \pm 3.8$ & $10.6 \pm 5.8$ \\
$\quad$ Month 6 & $6.3 \pm 3.4$ & $7.7 \pm 4.1$ \\
AZA dosage, mg/day & & \\
$\quad$ Month 2 & - & $43.9 \pm 26.9$ \\
$\quad$ Month 4 & - & $40.5 \pm 32.5$ \\
Month 6 & & $57.5 \pm 45.7$ \\
MMF dosage, mg/day & $1,697 \pm 462$ & - \\
$\quad$ Month 2 & $1,658 \pm 478$ & - \\
Month 4 & $1,614 \pm 512$ & - \\
$\quad$ Month 6 & & \\
MMF trough level, mg/l & $2.9 \pm 2.9$ & - \\
$\quad$ Month 2 & $3.3 \pm 3.2$ & - \\
Month 4 & $3.2 \pm 3.0$ & - \\
Month 6 & &
\end{tabular}

Table 3. Results of different surgeons responsible for the transplantations

\begin{tabular}{lllll}
\hline $\begin{array}{l}\text { Surgeon } \\
\text { No. }\end{array}$ & $\begin{array}{l}\text { Total KTx } \\
\mathrm{n}\end{array}$ & $\begin{array}{l}\text { Lymphoceles } \\
\mathrm{n} / \%\end{array}$ & $\begin{array}{l}\text { Symptomatic } \\
\text { lymphoceles } \\
\mathrm{n} / \%\end{array}$ & $\begin{array}{l}\text { Wound } \\
\text { infections } \\
\mathrm{n} / \%\end{array}$ \\
\hline 1 & 29 & $11 / 38$ & $1 / 3$ & $2 / 7$ \\
2 & 15 & $8 / 53$ & $3 / 20$ & $5 / 33$ \\
3 & 19 & $6 / 32$ & $2 / 11$ & $4 / 22$ \\
4 & 8 & $3 / 38$ & $1 / 13$ & $1 / 13$ \\
5 & 32 & $18 / 56$ & $7 / 22$ & $4 / 13$ \\
6 & 31 & $19 / 61$ & $7 / 23$ & $3 / 10$ \\
7 & 20 & $12 / 60$ & $2 / 10$ & $2 / 10$ \\
\hline
\end{tabular}

Table 4. Other potential risk factors for wound healing problems and lymphocele formation

\begin{tabular}{llll}
\hline & MMF group & AZA group & $\mathrm{p}$ \\
\hline $\begin{array}{l}\text { Serum creatinine, mg/dl } \\
\quad \text { Month 2 }\end{array}$ & & & \\
$\quad$ Month 4 & $1.74 \pm 1.05$ & $1.72 \pm 0.70$ & 0.14 \\
$\quad$ Month 6 & $1.73 \pm 0.97$ & $1.61 \pm 0.58$ & 0.13 \\
Body weight, kg & $1.74 \pm 1.28$ & $1.65 \pm 0.60$ & 0.16 \\
$\quad$ Month 2 & & & \\
$\quad$ Month 4 & $73.4 \pm 15.1$ & $72.0 \pm 13.9$ & 0.54 \\
Month 6 & $74.4 \pm 16.7$ & $73.9 \pm 14.6$ & 0.82 \\
& $76.3 \pm 13.7$ & $75.2 \pm 14.4$ & 0.99 \\
\hline
\end{tabular}

MMF and Wound Complications after KTx patient in the AZA group had to be drained twice) and 8 (MMF) versus 0 (AZA) sclerotherapies. In 1 or 2 patients operative intervention (in all cases a laparoscopic lymphocele fenestration with intraperitoneal drainage) had to be performed. None of these interventions were distributed differently between the groups. We did not observe any secondary complications of lymphocele treatment, no graft was lost due to the fluid collection or the performed interventions.

Altogether, 7 experienced urological surgeons were responsible for the 154 transplantations. Table 3 shows the results of each surgeon concerning every distinct endpoint. Surgeons differed in the number of transplantations performed and by the rates of complications but we could not detect any significant differences using $\chi^{2}$ testing.

\section{Graft Function, Diabetes and Other Risk Factors for Wound Complications}

Graft function measured as serum creatinine over time was not significantly different between both groups as summarized in table 4 . Body mass index (BMI) measured before transplantation was comparable between the groups. Both patient groups showed an increase in body weight of about $4 \%$ during the first 6 months after transplantation; however, neither body weight at the different time points nor the changes in body weight in the groups differed. Furthermore, incidences of diabetes mellitus before as well as after transplantation (posttransplant diabetes mellitus; PTDM) as classical risk factors for wound complications were comparable.

We conducted a series of univariate and multivariate log regression analyses concerning incidences of the primary endpoints total lymphoceles, symptomatic lymphoceles and wound infections. Lymphoceles in general were observed more often in patients receiving MMF than AZA; this was also strongly significant in the multivariate analysis. We saw a trend for lymphoceles depending on serum creatinine levels at all time points; however, this effect disappeared in multivariate testing (table 5). Considering only symptomatic lymphoceles, the negative effect of MMF was no longer statistically significant, but a rather strong effect of acute rejection episodes was found; patients without acute rejections tend to have more symptomatic lymphoceles than patients with an episode (table 6). We could not demonstrate any effect of the tested variables on wound infections in multivariate testing; the significant univariate dependency on age disappeared in the multivariate scenario (table 7). 
Table 5. Uni- and multivariate logistic regression analysis concerning the incidence of lymphoceles

\begin{tabular}{|c|c|c|c|c|c|c|}
\hline & \multicolumn{3}{|c|}{ Univariate } & \multicolumn{3}{|c|}{ Multivariate } \\
\hline & Exp. (B) & $95 \%$ CI & $\mathrm{p}$ & Exp. (B) & $95 \%$ CI & $\mathrm{p}$ \\
\hline Age per year & 0.999 & $0.97-1.02$ & 0.94 & & & \\
\hline Female gender & 1.12 & $0.58-2.13$ & 0.74 & & & \\
\hline Induction vs. no induction & 1.21 & $0.60-2.42$ & 0.60 & & & \\
\hline MMF vs. AZA & 2.02 & $1.16-4.19$ & 0.016 & 2.64 & $1.09-6.41$ & 0.032 \\
\hline ARE vs. no ARE & 0.73 & $0.38-1.38$ & 0.33 & & & \\
\hline DM vs. no DM pre-KTx & 0.63 & $0.24-1.64$ & 0.34 & & & \\
\hline PTDM vs. no PTDM & 1.15 & $0.55-3.00$ & 0.79 & & & \\
\hline BMI before KTx per unit & 1.04 & $0.95-1.12$ & 0.41 & & & \\
\hline S-Crea month 2 & 1.52 & $0.96-2.41$ & 0.072 & 1.86 & $0.76-4.56$ & 0.18 \\
\hline S-Crea month 4 & 1.54 & $0.93-2.55$ & 0.097 & 1.17 & $0.28-4.87$ & 0.83 \\
\hline S-Crea month 6 & 1.39 & $0.87-2.21$ & 0.168 & 0.85 & $0.27-2.68$ & 0.78 \\
\hline
\end{tabular}

$\mathrm{ARE}=$ Acute rejection episode; $\mathrm{DM}=$ diabetes mellitus.

Table 6. Uni- and multivariate logistic regression analysis concerning the incidence of symptomatic lymphoceles

\begin{tabular}{|c|c|c|c|c|c|c|}
\hline & \multicolumn{3}{|c|}{ Univariate } & \multicolumn{3}{|c|}{ Multivariate } \\
\hline & Exp. (B) & $95 \% \mathrm{CI}$ & $\mathrm{p}$ & Exp. (B) & $95 \% \mathrm{CI}$ & $\mathrm{p}$ \\
\hline Age per year & 1.02 & $0.98-1.05$ & 0.39 & & & \\
\hline Female gender & 0.65 & $0.27-1.59$ & 0.35 & & & \\
\hline Induction vs. no induction & 1.57 & $0.63-3.90$ & 0.33 & & & \\
\hline MMF vs. AZA & 2.26 & $0.91-5.65$ & 0.081 & 2.10 & $0.66-6.71$ & 0.21 \\
\hline ARE vs. no ARE & 0.23 & $0.08-0.72$ & 0.011 & 0.19 & $0.05-0.68$ & 0.011 \\
\hline DM vs. no DM pre-KTx & 0.57 & $0.12-2.61$ & 0.47 & & & \\
\hline PTDM vs. no PTDM & 1.21 & $0.42-3.52$ & 0.73 & & & \\
\hline BMI before KTx per unit & 1.01 & $0.92-1.10$ & 0.68 & & & \\
\hline S-Crea month 2 & 1.19 & $0.78-1.81$ & 0.42 & & & \\
\hline S-Crea month 4 & 1.31 & $0.83-2.08$ & 0.24 & & & \\
\hline S-Crea month 6 & 1.36 & $0.92-2.00$ & 0.12 & & & \\
\hline
\end{tabular}

$\mathrm{ARE}=$ Acute rejection episode; $\mathrm{DM}=$ diabetes mellitus.

\section{Discussion}

Postoperative surgical complications after kidney transplantation have been considerably reduced in the last years due to improvements in surgical technique and immunosuppressive therapy [2]. However, these complications still remain a cause of significant morbidity, prolonged hospital stays, readmissions and increased costs of the transplant procedure [8]. Symptomatic lymphoceles are the most common fluid accumulations following kidney transplantation and are seen in $0.5-22 \%$ of trans- planted patients [9]. More than $95 \%$ of them appear in the first 6 months after KTx. Reasons for their appearance are seen in the incomplete ligation of lymphatic vessels at the hilus of the graft and around the iliac vessels of the recipient. As potential risk factors, retransplantations, acute rejection episodes, acute tubular necrosis, high steroid doses and former graft biopsies are considered [10]. Fluid accumulations can cause harm by graft displacement and compression of the ureter, the bladder or the graft vessels with consequent graft dysfunction and by the compression of the iliac vessels with the possibility of 
Table 7. Uni- and multivariate logistic regression analysis concerning the incidence of wound infections

\begin{tabular}{|c|c|c|c|c|c|c|}
\hline & \multicolumn{3}{|c|}{ Univariate } & \multicolumn{3}{|c|}{ Multivariate } \\
\hline & Exp. (B) & $95 \% \mathrm{CI}$ & $\mathrm{p}$ & Exp. (B) & $95 \% \mathrm{CI}$ & $\mathrm{p}$ \\
\hline Age per year & 1.05 & $1.01-1.09$ & 0.027 & 1.03 & $0.99-1.08$ & 0.13 \\
\hline Female gender & 0.66 & $0.26-1.67$ & 0.38 & & & \\
\hline Induction vs. no induction & 0.53 & $0.17-1.67$ & 0.28 & 0.53 & $0.13-2.19$ & 0.38 \\
\hline MMF vs. AZA & 0.57 & $0.22-1.47$ & 0.24 & 0.82 & $0.24-2.81$ & 0.75 \\
\hline ARE vs. no ARE & 1.33 & $0.53-3.35$ & 0.55 & & & \\
\hline DM vs. no DM pre-KTx & 2.46 & $0.79-7.68$ & 0.122 & & & \\
\hline PTDM vs. no PTDM & 2.69 & $1.03-7.01$ & 0.043 & 1.87 & $0.46-7.52$ & 0.38 \\
\hline BMI before KTx per unit & 1.10 & $0.98-1.23$ & 0.17 & 1.07 & $0.95-1.22$ & 0.27 \\
\hline S-Crea month 2 & 1.35 & $0.46-3.96$ & 0.59 & & & \\
\hline S-Crea month 4 & 0.75 & $0.10-5.43$ & 0.78 & & & \\
\hline S-Crea month 6 & 0.68 & $0.13-3.82$ & 0.68 & & & \\
\hline
\end{tabular}

$\mathrm{ARE}=$ Acute rejection episode; $\mathrm{DM}=$ diabetes mellitus

leg edema or even deep venous thrombosis [9]. Wound complications can be categorized as dehiscences, seromas and other fluid collections as well as superficial and deep infections [2]. Risk factors for wound complications include obesity, older age, diabetes mellitus, alcohol and nicotine abuse, malnutrition and immunosuppressive agents [11].

In the last decade an array of new immunosuppressive drugs received approval for maintenance immunosuppression after kidney transplantation. Some of these newer substances provoke distinctive side effects not seen on the conservative regimens used before. This was especially true for sirolimus, the mTOR inhibitor, which led to a considerable rise in wound healing problems and lymphocele formation [20]. Another of these newer substances is mycophenolate mofetil, marketed in the EU since 1995. MMF is known to be a potent immunosuppressive agent [12] with the potential to improve long-term patient and graft survival $[13,14]$. MMF, a prodrug of mycophenolic acid (MPA), blocks the purine synthesis by inhibiting the rate limiting enzyme inosine monophosphate dehydrogenase (IMPDH). The result is an inhibition of $\mathrm{B}$ and $\mathrm{T}$ lymphocyte proliferation, a reduction of antibody synthesis and a decrease of the migration of inflammatory cells into tissues after antibody binding [15]. MPA leads to a five times more effective inhibition of IMPDH type II, which is mainly expressed in activated lymphocytes, but it also inhibits the type I isoform expressed in most other cell types [16]. This may also cause a dose-dependent inhibition of fibroblast proliferation, as has been shown in rat kidneys [17], cultures of human Tenon cells of the eye [18] as well as in dermal fibroblast cultures [19]. A group from Heidelberg reported recently that MPA administration to cultured human fibroblasts resulted in a downregulation of cytoskeletal proteins, a rearrangement of the cytoskeleton and impaired migrational capacity. These results could also be replicated in skin biopsies of transplant patients [21]. Therefore, an impairment of healing processes, for example of separated tissues or disrupted lymphatic vessels, seems principally possible.

Also in our center we had the impression of increasing wound problems since the introduction of MMF in our routine regime. Therefore, we performed this retrospective analysis of two well-matched patient cohorts receiving either MMF or AZA together with CsA as maintenance immunosuppression in respect of impaired wound healing.

Studies on these types of side effects of MMF are relatively scarce in the literature. There is only one retrospective report addressing risk factors for wound infections with MMF. Humar et al. [7] evaluated data of more than 2,000 kidney recipients transplanted between 1984 and 1998. Wound infections were reported in only 97 of these patients (4.8\%). The authors found that MMF application was one of the risk factors for wound infections $(\mathrm{RR}=2.4)$ and hernias or dehiscences ( $R R=3.5)$; other significant factors were obesity, diabetes, reoperations through the same wound and urine leakage. The group considered the higher immunosuppressive load (in comparison to AZA) as causal for this outcome. A second analysis was done by 
Kuppahally et al. [22] in heart-transplanted patients. This group retrospectively compared postsurgical wound complications in patients on irolimus versus MMF as first-line therapy. They found wound complications in $28 \%$ of all MMF-treated patients which was significantly less than in the comparator group. The authors could not demonstrate any other classical risk factor for wound problems in their population.

Considering postsurgical wound infections, we cannot endorse these results in our KTx population. First of all we observed wound problems in about $14 \%$ of all cases, being slightly more frequent in patients treated with MMF. However, none of the potential risk factors considered to be crucial appeared to be influential in our analysis. From our data, we could not substantiate any influence of the intake of MMF on the occurrence of wound infections. Taking these reports together, incidence rates differed significantly between the publications, most likely due to diverse populations, eras and also differing definitions for this kind of complication in different centers and/or registries; therefore, a unified result cannot be extracted.

Regarding lymphatic fluid accumulations we found a different pattern. In our population we could demonstrate a significant increase in sonographically detectable lymphoceles in the group receiving MMF. The frequency of lymphoceles seems rather high in comparison to other sources, but contrary to other studies we counted every fluid collection irrespective of its clinical significance. There was also a trend for more symptomatic lymphoceles in need of more drainage procedures, sclerotherapies or reoperations in the MMF group. Fortunately, none of these symptomatic cases resulted in graft loss.

Patients with symptomatic lymphoceles had less episodes of acute rejections and therefore also less steroid pulses, but the cumulative steroid dose was comparable between the groups. This association remained significant even after adjustment for other risk factors; a reason for this discordance to other publications could not be detected.

Also other explanations for the increased incidence of lymphoceles remain speculative. We did not change or increase our in- and out-patient surveillance in the observation period, the diagnostic routine described in $\mathrm{Pa}$ tients and Methods was applied to every patient described in here. Therefore, an artificial increase in lymphocele frequency due to increased monitoring over time seems unlikely. Another reason for our finding could be a change in surgical procedure or the composition of the surgical teams; however, no alternations concerning both aspects were noted in the relevant era. Other classical risk factors for lymphocele formation despite acute rejections were not more frequent in the MMF group [10]. We did not observe significant differences in recipient risk factors such as age, obesity, diabetes before or after KTx or number of retransplants. We do not routinely collect information on acute tubular necrosis incidence, graft biopsy incidence, substance abuse or malnutrition; therefore, the attributable risk to these recipient factors could not be calculated. In multivariate analysis only MMF persisted as variable with increased hazard for lymphocele formation but not for symptomatic lymphoceles with the need for intervention.

There are several limitations to the present study. The study is retrospective in nature and even if significant, reporting bias is very much reduced by prospective data collection by only two responsible persons. Our findings nevertheless require confirmation in a prospective trial. Patient numbers are quite small. Readers must keep in mind that the results are also only representative for $\mathrm{Eu}-$ ropean or Caucasian populations. Both groups differed in the use of induction therapies; however, no increase in lymphoceles or wound healing difficulties due to antiCD25 treatment was ever documented. Due to the otherwise homogeneous maintenance immunosuppression, we consider the different period of transplantation between the groups as not relevant.

Taking our observations together, we can show for the first time that MMF administration after kidney transplantation appears to result in more lymphoceles without an impairment of graft function or graft survival. Therefore, in case of early MMF administration post-transplantation potential risk factors for lymphocele formation should be avoided as far as possible, and meticulous preparation of the lymphatic vessels, in the hilus of the donor organ as well as around the recipient vessels, is imperative.

\section{Conflicts of Interest}

K. Lopau received travelling grants from Roche Pharma. 


\section{References}

1 Oniscu GC, Brown, H, Forsythe JLR: Impact of cadaveric renal transplantation on survival in patients listed for transplantation. J Am Soc Nephrol 2005; 16:1859-1865.

$\checkmark 2$ Mehrabi A, Fonouni H, Wente M, et al: Wound complications following kidney and liver transplantation. Clin Transplant 2006; 20(suppl 17):97-110.

\3 Hernández D, Rufino M, Armas S, et al: Retrospective analysis of surgical complications following cadaveric kidney transplantation in the modern transplant era. Nephrol Dial Transplant 2006;21:2908-2915.

4 Ghasemian SMR, Guleria AS, Khawand NY, Light J: Diagnosis and management of the urologic complications of renal transplantation. Clin Transplant 1996;10:218-223.

5 van Roijen JH, Kirkels WJ, Zietse R, Roodnat JI, Weimar W, Ijzermans JN: Long-term graft survival after urological complications of 695 kidney transplantations. J Urology 2001;165:1884-1887.

6 Cohan RH, Saeed M, Sussman SK, et al: Percutaneous drainage of pelvic lymphatic fluid collections in the renal transplant patient. Invest Radiol 1987;22:864-867.

7 Humar A, Ramcharan T, Denny R, Gillingham KJ, Payne WD, Matas AJ: Are wound complications after a kidney transplant more common with modern immunosuppression? Transplant 2001;72:1920-1923.

-8 Johnson CP, Kuhn EM, Hariharan S, et al: Pre-transplant identification of risk factors that adversely affect length of stay and charges for renal transplantation. Clin Transplant 1999; 13:168-175.
-9 Langer RM, Kahan BD: Incidence, therapy, and consequences of lymphocele after sirolimus-cyclosporine-prednisone immunosuppression in renal transplant patients. Transplant 2002;74:804-808.

10 Khauli RB, Stoff JS, Lovewell T, et al: Post transplant lymphoceles: a critical look into the risk factors pathophysiology and management. J Urol 1993;150:22-26.

11 Stephan RN, Munschauer CE, Kumar MS: Surgical wound infection in renal transplantation: outcome data in 102 consecutive patients without perioperative systemic antibiotic coverage. Arch Surg 1997;132:1315-1318

12 Halloran P, Mathew T, Tomlanovich S, et al: Mycophenolate mofetil in renal allograft recipients: a pooled efficiency analysis of three randomized, double-blind, clinical trials in preventing rejection. The International Mycophenolate Mofetil Renal Transplant Study Groups. Transplant 1997;63:39-47.

13 Ojo AO, Meier-Kriesche HU, Hanson JA, et al: Mycophenolate mofetil reduces late renal allograft loss independent of acute rejection. Transplant 2000;69:2405-2409.

14 Meier-Kriesche HU, Steffen BJ, Hochberg AM, et al: Mycophenolate mofetil versus azathioprine therapy is associated with a significant protection against long-term renal allograft function deterioration. Transplant 2003;75:1341-1346.
15 Allison AC, Eugui EM: Mycophenolate mofetil and its mechanism of action. Immunopharmacology 2000;47:85-118.

16 Carr SF, Papp E, Wu JC, Natsumeda Y: Characterization of human type I and type II IMP dehydrogenases. J Biol Chem 1993;268: 27286-27290

17 Badid C, Vincent M, McGregor B, et al: Mycophenolate mofetil reduces myofibroblast infiltration and collagen III deposition in the rat remnant kidney. Kidney Int 2000;58:5161.

18 Heinz C, Hudde T, Heise K, Steuhl KP: Antiproliferative effect of mycophenolate mofetil on cultured human Tenon fibroblasts. Graefes Arch Clin Exp Ophtalmol 2002;240:408414.

19 Morath C, Zeier M: Review of the antiproliferative properties of mycophenolate mofetil in non-immune cells. Int J Pharmacol Ther 2003;41:465-469.

20 Knight RJ, Villa M, Laskey R, et al: Risk factors for impaired wound healing in irolimustreated renal transplant recipients. Clin Transplant 2007;21:460-465.

21 Morath C, Reuter H, Simon V, et al: Effects of mycophenolic acid on human fibroblast proliferation, migration and adhesion in vitro and in vivo. Am J Transplant 2008;8: 1786-1797.

$>22$ Kuppahally S, Al-Khaldi A, Weisshaar D, et al: Wound healing complications with de novo irolimus versus mycophenolate mofetil-based regimen in cardiac transplant recipients. Am J Transplant 2006;6:986-992. 